\title{
Automatic Liver Segmentation Method on Contrast-Enhanced MR Imaging Using Probabilistic Atlas
}

\author{
Yuriko Yoshino a,, , Hideki Honda ${ }^{\mathrm{a}}$, Hyoungseop Kimª, Joo Kooi Tan, Seiji Ishikawa ${ }^{\mathrm{a}}$ \\ Seiichi Murakami ${ }^{\mathrm{b}}$, Guangxu $\mathrm{Li}^{\mathrm{c}}$ \\ aDepartment of Control Engineering Kyushu Institute of Technology, 1-1, Sensui-cho, Tobata-ku, Kitakyushu, \\ 804-8550, Japan \\ bepartment of Radiology University of Occupational and Environmental Health, 1-1, Ishigaoka, Yahata-nishi-ku, \\ Kitakyushu, 807-8555, Japan \\ 'Tianjin Polytechnic University, No.399, Binshui Xi Road, Xiqing District, Tianjin, 300387, China \\ *kimhs@cntl.kyutech.ac.jp
}

\begin{abstract}
In medical field, computer aided diagnosis (CAD) system such as a system which can diagnosis multi-organ multi-disease has been expected. In implementing the system, automatic liver segmentation on abdominal MR images is one of the most important steps. However, precise automatic liver segmentation on MR images remains a major challenge since there are differences of organ shape and intensity distribution in each MR images. It is necessary to estimate these appropriately. In this paper, we present an automatic segmentation method with consideration of intensity and statistical model. Namely, we estimate probabilistic atlas and incorporate it into region growing method. We have carried out experiments by the proposed method on 7 cases of abdominal contrast-enhanced magnetic resonance (MR) Images. The experimental results show that the performance of the method using region growing with probabilistic atlas is higher than that without probabilistic atlas.
\end{abstract}

Keywords: contrast-enhanced MR image, probabilistic atlas, liver segmentation, region growing method.

\section{Introduction}

In medical field, computer aided diagnosis (CAD) system to improve performance and accuracy in visual screening. Also quality of life (QOL) of patients by early detection has received broad attention. The CAD system in some field such as mammography and thoracic multi detector computed tomography (MDCT) screening has been implemented and put to practical use ${ }^{(1,2)}$. In recent years, alternative CAD system which can diagnose multi-organ or multi-disease to conventional CAD which can only diagnosis single-organ or single-disease has been focused ${ }^{(3)}$. Modelling of anatomical structures and segmentation processing are required in implementation on CAD system for multi-organ or multi-disease. Segmentation of organs on MDCT images and MR images is required in diagnosis assist such as analytics of organ structure, detection of disease, diagnosis of benign and malignant, and affects accuracy of these processes. In recent years, techniques applying statistical atlas such as probabilistic atlas and statistical shape model have been actively studied. In the studies by Kamber et al. ${ }^{(4)}$ and Leempnt et al. ${ }^{(5)}$, probabilistic atlas is applied in segmentation of brain MR images. Probabilistic atlas has begun to be used in segmentation of abdominal organs since 2000s. In the study by Okada et al. ${ }^{(6)}$, the segmentation of livers on computed tomography (CT) images using statistical shape model and probabilistic atlas is proposed. However, precise automatic liver segmentation on MR images remains a major challenge since there are differences of organ shape and intensity distribution in each MR images. In this paper, to improve the segmentation accuracy we present an automatic segmentation method with consideration of intensity and statistical model. In our proposed method, probabilistic atlas is estimated and incorporated into region growing method. In the experimental results, we performed 7 cases of abdominal contrast-enhanced MR images and satisfactory results are obtained. 


\section{Method}

The Main process is shown in Fig. 1. We calculate probabilistic atlas using training MR images before the first step of the main process. The step after normalization of an input image is to extract a liver region roughly by the threshold technique which is calculated by discriminant analysis method ${ }^{(7)}$. The next step is to match between the extracted region and probabilistic atlas using distance transformation as described in subsection 2.1. The final step is to extract the liver precisely by region growing method incorporating the probabilistic atlas as described in subsection 2.2.

\subsection{Registration by probabilistic atlas}

Here, probabilistic atlas is calculated using training MR images which are normalized in volume and matched to them by affine transformation. In this paper, the parameter of affine transformation which maximizes dice similarity coefficient (DSC) is estimated by particle swarm optimization (PSO) ${ }^{(8)}$. DSC is given by the equation (1).

$$
D S C=2 \times \frac{R(A \cap B)}{R(A)+R(B)}
$$

where $A, B$ are labels. $R(A), R(B)$ represent the number of labeled pixels, respectively. The DSC ranges from 0 to 1 . If overlapped pixels of the two labels are increased, the DSC approaches 1. PSO is a global search method based on simulation of social behavior and also an algorithm modeling the movement of bird flocks. In the PSO, particles which are individuals of a swarm migrate repeatedly to better positions in a search space. A particle repositions in the search space under the affection of the best position in previous positions of the particle and that of all particles. Suppose $\mathbf{x}_{i}(t)$ denotes the position of particle $i$ at the $t$-th generation, pbest $(t, i)$ denote the best position at previous generations of $\mathbf{x}_{i}$ and $\operatorname{gbest}(t)$ denotes the best position at previous generations in the whole swarm, its velocity $\mathbf{v}_{\mathbf{i}}(t+1)$ is updated by the following equations.

$$
\begin{aligned}
& \mathbf{V}_{i}(t+1)=w \mathbf{V}_{i}(t)+\eta_{1} \times r \times\left(\text { pbest }(t, i)-\mathbf{X}_{\mathbf{i}}(t)\right) \\
& +\eta_{2} \times R \times\left(\operatorname{gbest}(t)-\mathbf{X}_{\mathbf{i}}(t)\right) \\
& \mathbf{X}_{\mathbf{i}}(t+1)=\mathbf{X}_{\mathbf{i}}(t)+\mathbf{V}_{\mathbf{i}}(t+1)
\end{aligned}
$$

where, $w$ is the inertia weight. $\eta_{1}$ and $\eta_{2}$ are acceleration constants, respectively. $r$ and $R$ are random real numbers between the range $[0,1]$. In this paper, at the first step, a swarm of particles each of which represents affine parameters is formed. The parameters are randomly generated under uniform distribution. At the second step, the DSC of each paricles is calculated and pbest, gbest is updated. After that, each particle is repositioned by Eq. (2a) and Eq. (2b). The processes after the second step is iterated until termination condition, where gbest is the optimal solution.

Existence probability at a position whose coordinate is $(x, y, z)$ is represented as $p(x, y, z)$ using window function as following equation (3).

$$
\begin{aligned}
& p(x, y, z) \\
& =\frac{1}{N} \sum_{d x} \sum_{d y} \sum_{d z} w(d x, d y, d z) \\
& \sum_{i=1}^{N} b_{i}(x+d x, y+d y, z+d z)
\end{aligned}
$$

where, $N$ is number of training MR images, $w$ represents window function, $b_{i}$ is 1 when the position is inside organs, elsewhere is 0 , respectively. In this paper, we use Gaussian function as window function ${ }^{(9)}$. Then, existence probability is normalized to be in the range $[0,1]$. We define this as probabilistic atlas. In the next step, the input image is normalized in volume, and its liver region is extracted roughly by the threshold calculated by discriminant analysis $\operatorname{method}^{(7)}$. The small holes such as a part of blood vessels and lesions in liver region are filled by closing and opening in morphologic operation. The region after rough extraction includes over-extraction regions which prevent precise registration to the probabilistic atlas. Therefore, we calculate distance transformation images respectively from

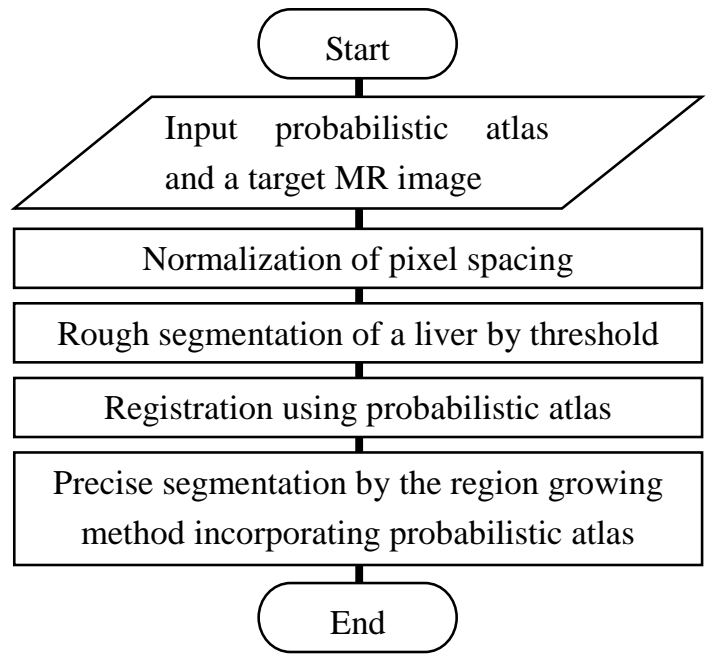

Fig. 1. The overall algorithm of the proposed method. 
the input image, probabilistic atlas and remove regions whose distance transformation value is lower than the proper threshold, focusing on the thing that liver is the largest in abdominal organs. The parameter of transformation in registration is estimated by these distance transformation images, and the target input image and the probabilistic atlas are matched by it. Moreover, in the probabilistic atlas image, we define the region whose pixels have higher existence probability than the fixed value as the average shape. And the average shape and the liver region extracted roughly are matched finally by estimating the transform parameter. The result of probabilistic atlas calculated by Eq. (1) is showed in Fig. 2. A slice of the probabilistic atlas is showed in Fig. 2(a). It is indicated that the closer to white the color of a pixel is, the higher existence probability is. The average shape existence probability of which is more than $50[\%]$ is showed by volume rendering in Fig. 2(b). The registration process is showed in Fig. 3. The target input image is showed in Fig. 3(a). The result of rough extraction is showed in Fig. 3(b). Distance transformation image is showed in Fig. 3(c). The center of the river is estimated using distance transformation and thresholding as shown in Fig. 3(d). The parameter of transformation in registration is estimated using the center. The result of registration is shown in Fig. 3(e). The red region is the probabilistic atlas matched to the river on the input image.

\subsection{Precise extraction by region growing method}

The seed point of the proposed region growing method is the pixel whose intensity is higher than the threshold and value of distance transformation is the highest in the input image. We define the equation (4a) and (4b) as the condition of the proposed region growing which incorporates probabilistic atlas.

$$
\begin{aligned}
& d v(x, y, z) \leq \alpha \times p(x, y, z)+(1-\alpha) \times \beta \\
& \gamma \leq v(x, y, z)
\end{aligned}
$$

where, $v(x, y, z), d v(x, y, z)$ and $p(x, y, z)$ are intensity, difference of intensity to a neighboring pixel and a probabilistic atlas respectively. $\alpha, \beta$ and $\gamma$ are weight for probabilistic atlas, constant and the threshold calculated by discriminant analysis $\operatorname{method}^{(7)}$ respectively. The strict condition of connected components to pixels each of which has a low existence probability is given by Eq. (4a), and over-connection to a low intensity region is prevented by Eq. (4b). After applying region growing, the small holes are filled in finally.

\section{Experimental results}

To illustrate the performance of our proposed scheme, we have carried out experiments by the proposed method on 7 cases of abdominal contrast-enhanced MR images. The image information are shown in the Table 1 . The segmentation applying probabilistic atlas $(\alpha=0.5)$ and not applying probabilistic atlas $(\alpha=0.0)$ have been respectively executed and these results were compared. Accuracy of segmentation were evaluated using true positive (TP), false positive (FP) and degree of correspond as following the equation (5), (6) and (7) respectively.

$$
\begin{aligned}
& T P=\frac{n(A \cap B)}{n(A)} \times 100 \\
& F P=\frac{n(C)}{n(B)} \times 100 \\
& I=\frac{n(A \cap B)}{n(A \cup B)} \times 100
\end{aligned}
$$

where $A, B$ and $C$ represent the correct region, segmentation result and over-extraction region respectively. $I$ is degree of correspond. $n$ represents number of pixels in a region. The evaluation values in $\alpha=0.5$ and $\alpha=0.0$ are shown in Table 2 and Table 3 respectively. The one of the results in $a=0.5$ and $a=0.0$ are shown in Fig. 4 and Fig. 5, (a), (b), (c), (d) of which show 80-th, 100-th, 120-th, 140-th slices respectively. The red line draws the result by the proposed method, the green line draws the correct contour of the liver and they overlap in the yellow line. In Table 2 and Table 3 ,

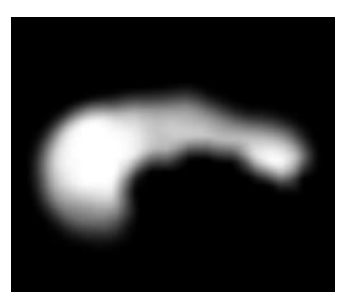

(a) A slice of the probabilistic atlas

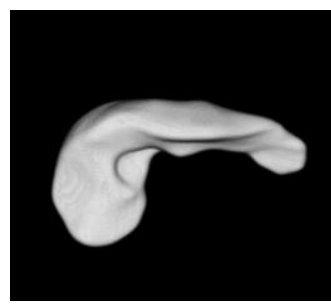

(b) The average shape by volume rendering

Fig. 2. The result of the calculated probabilistic atlas. 


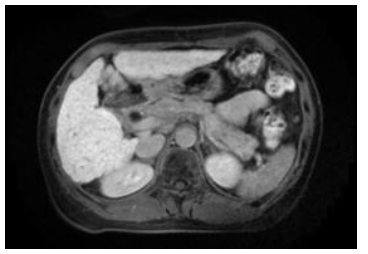

(a) An input image
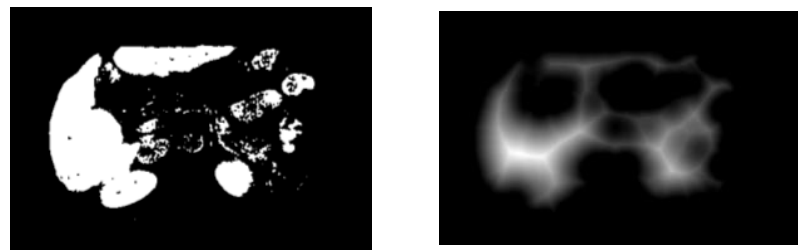

(b) The result of (c) The result of distance thresholding by transformation discriminant analysis ${ }^{(7)}$

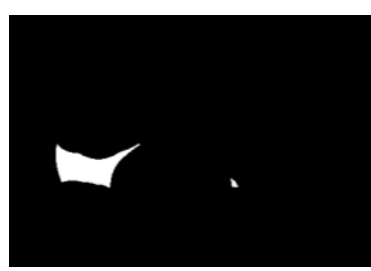

(d) The result of thresholding to the distance transformation image

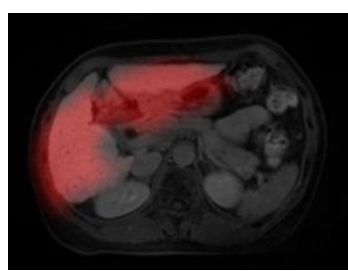

(e) The result of registration probabilistic atlas between the river and the

Fig. 3. The result of rough segmentation, distance transformation and registration between the input image and probabilistic atlas.

FP in $\alpha=0.5$ is lower than that in $\alpha=0.0$ in all cases, and the average of degree of correspond in $\alpha=0.5$ is higher than that in $\alpha=0.0$. A comparison of Fig. 4 and Fig. 5 shows that the over-extraction region in $\alpha=0.5$ is smaller than that in $\alpha=0.0$. On the other hand, TP in $\alpha=0.5$ is lower than that in $\alpha=0.0$ in all cases. Since FP is lower in the case applying probabilistic atlas, it is indicated that over-extraction is prevented using probabilistic atlas. This is due to restraint of over-extraction to organs around a liver by the restrict condition of connected components to pixels which have low existence probability. On the other hand, $\mathrm{TP}$ is lower in the case applying probabilistic atlas, too. This is caused by lack of extraction near contour of a liver. Since liver position is not considered in estimation of probabilistic atlas and insufficient registration to a target liver is caused due to it, pixels near the contour have low probabilistic atlas as shown in Fig. 6. Fig. 6(a) shows the
Table 1. Image information

\begin{tabular}{|c|c|}
\hline Image size [pixel] & $512 * 512$ \\
\hline Pixel size $[\mathrm{mm}]$ & 0.742 \\
\hline Slice thickness $[\mathrm{mm}]$ & 2.0 \\
\hline
\end{tabular}

Table 2. TP, FP and degree of correspond in $\alpha=0.5$

\begin{tabular}{|c|c|c|c|}
\hline Case number & TP & FP & $\begin{array}{c}\text { Degree of } \\
\text { correspond }\end{array}$ \\
\hline 1 & 89.03 & 5.91 & 84.32 \\
\hline 2 & 95.20 & 17.50 & 79.21 \\
\hline 3 & 83.83 & 6.29 & 79.36 \\
\hline 4 & 95.06 & 21.62 & 75.31 \\
\hline 5 & 65.70 & 7.63 & 62.32 \\
\hline 6 & 91.80 & 6.91 & 85.95 \\
\hline 7 & 93.22 & 14.67 & 80.35 \\
\hline Average & 87.69 & 11.50 & 78.12 \\
\hline
\end{tabular}

Table 3. TP, FP and degree of correspond in $\alpha=0.0$

\begin{tabular}{|c|c|c|c|}
\hline Case number & TP & FP & $\begin{array}{c}\text { Degree of } \\
\text { correspond }\end{array}$ \\
\hline 1 & 96.38 & 19.09 & 78.53 \\
\hline 2 & 98.03 & 53.15 & 46.41 \\
\hline 3 & 95.07 & 8.99 & 86.91 \\
\hline 4 & 98.23 & 50.59 & 48.97 \\
\hline 5 & 94.25 & 19.47 & 76.76 \\
\hline 6 & 97.28 & 10.45 & 87.36 \\
\hline 7 & 97.31 & 21.13 & 77.18 \\
\hline Average & 96.65 & 26.12 & 71.73 \\
\hline
\end{tabular}

result of the registration between the probabilistic atlas and the input image and Fig. 6(b) shows the result of segmentation. For this reason pixels near contour of a liver are not connected.

\section{Conclusion}

In this paper, we have presented the segmentation method for a liver on a MR image using probabilistic atlas and region growing method incorporating it. Compared to the averages of FP and degree of correspond in the case not applying probabilistic atlas, the average of FP is lower and the average of degree of correspond is higher in the case applying probabilistic atlas. Therefore the result of this study reveals that our proposed method has capability to improve accuracy of segmentation and reduce over-extraction. However lack of extraction was occurred 


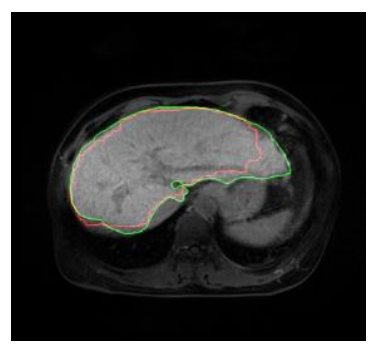

(a) 80th

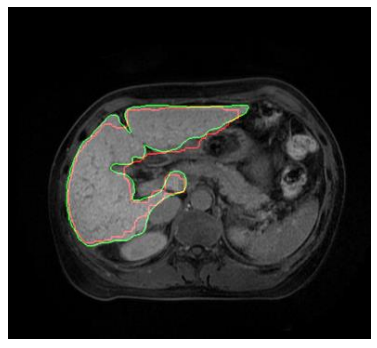

(c) 120 th

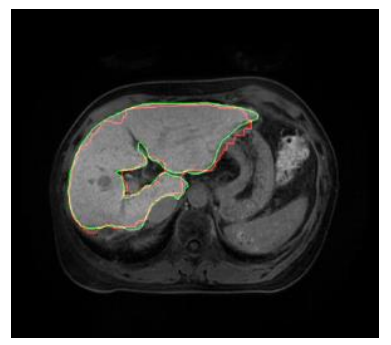

(b) 100 th

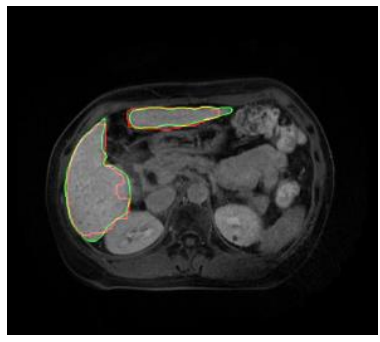

(d) 140th

Fig. 4. The result of segmentation in $\alpha=0.5$

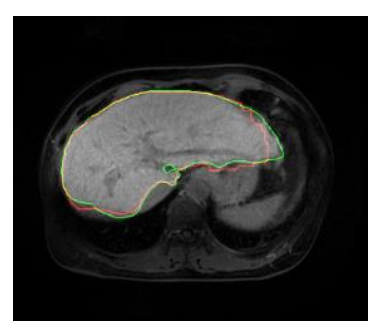

(a) 80th

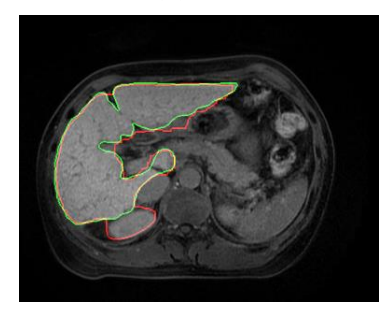

(c) 120 th

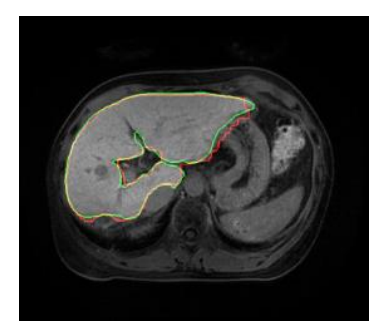

(b) 100 th

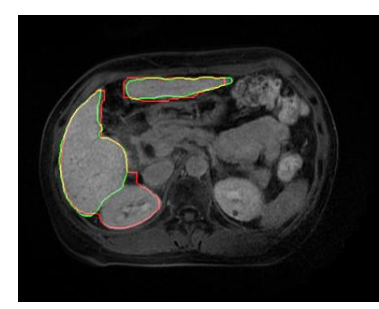

(d) 140 th

Fig. 5. The result of segmentation in $\alpha=0.0$

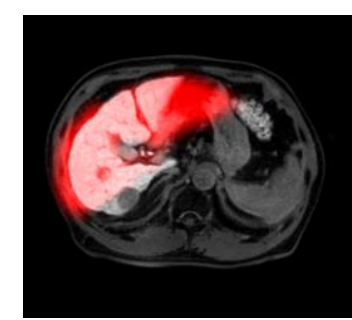

(a) The result of registration between the input image and the probabilistic atlas

Fig. 6. Experimental results of registration and segmentation. near the contour of a liver in some experimental results applying probabilistic atlas. In our future work, to further archive precise segmentation of a liver on a MR image, we improve the estimation method of probabilistic atlas and the registration method between probabilistic atlas and a target liver. Moreover, we aim to develop segmentation method using modelling organs around a liver to considerate relative positions of the organs.

\section{References}

(1) Roehrig, J., Doi, T., Hasegawa, A. et al. : "Clinical results with R2 Imagechecker system", Digital Mammography, Vol. 13, pp. 395-400, 1998

(2) Mitsubishi Space Software co.,ltd, Truedia, http://www.mss.co.jp/product/truedia.html (October 30, 2015 access)

(3) Kobatake, H. : "Future CAD in multi-dimensional Medical Images - Project on multi-organ, multi-disease CAD system", Computerized Medical Imaging and Graphics, Vol. 31, No. 4-5, pp. 258-266, 2007

(4) Kamber, M., Shinghal, R., Collins, D. L. et al. : "Model-based 3-D segmentation of multiple sclerosis lesions in magnetic resonance brain images", IEEE Trans. MI, Vol. 14, No. 3, pp. 442-453, 1995

(5) Leempnt, K. V., Maes, F., Vandermeulen, D. et al. : "Automated model-based tissue classification of MR images of the brain", IEEE Trans. MI, Vol. 18, No. 10, pp. 897-908, 1999

(6) Okada, T., Shimada, R., Sato, Y. et al. : "Automated segmentation of the liver from 3D CT images using probabilistic atlas and multi-level statistical shape model", Medical Image Computing and Computer-Assisted Intervention, pp. 86-93, 2007

(7) Otsu, N. : "A threshold selection method from gray-level histograms", IEEE Trans. SMC, Vol. 9, No. 1, pp. 62-66, 1979

(8) Kennedy, J. and Eberhart, R. : "Particle swarm optimization", Proc. IEEE Int. Conf. Neural Network, Vol. 4, pp. 1942-1948, 1995

(9) Shimizu, A., Ohno, R., Ikegami, T. et al. : "Segmentation of multiple organs in non-contrast 3D abdominal CT images", International Journal of Computer Assisted Radiology and Surgery, Vol. 2, No. 3, pp. 135-142, 2007 